\title{
Analysis of the Diagnosis and Treatment of Recurrent Rupture and Hemorrhage of Ovarian Corpus Luteum Cyst Induced by Imatinib
}

\author{
Huan Xia ${ }^{1}$, Weiwei Huang ${ }^{1}$, Yang Song ${ }^{1}$, Wenying Liu ${ }^{1}$, Huajuan Dong ${ }^{1}$, Li Qiu ${ }^{1}$, \\ Jiming Chen ${ }^{2, *}$ \\ ${ }^{1}$ Department of Obstetrics and Gynecology, Zigong Fourth People's Hospital, Zigong, China \\ ${ }^{2}$ Department of Obstetrics and Gynecology, the Affiliated Changzhou No. 2 People's Hospital of Nanjing Medical University, Changzhou, \\ China
}

Email address:

cjming@126.com (Jiming Chen)

${ }^{*}$ Corresponding author

\section{To cite this article:}

Huan Xia, Weiwei Huang, Yang Song, Wenying Liu, huajuan Dong, Li Qiu, Jiming Chen. Analysis of the Diagnosis and Treatment of Recurrent Rupture and Hemorrhage of Ovarian Corpus Luteum Cyst Induced by Imatinib. Journal of Gynecology and Obstetrics. Vol. 9, No. 4, 2021, pp. 100-103. doi: 10.11648/j.jgo.20210904.11

Received: June 11, 2021; Accepted: July 5, 2021; Published: July 10, 2021

\begin{abstract}
Objective: Rupture and hemorrhage of corpus luteum cyst is one of the common acute abdominal diseases in gynecology, which occurs in the middle and late menstrual cycle, and is often seen in women of childbearing age, often under the inducement of exercise and sexual life. There are few reports about drug-induced rupture and hemorrhage of corpus luteum cyst. Chronic myeloid leukemia (CML) is a common hematological disease and a chronic clonal myeloproliferative disease. Imatinib is a first-line drug for the treatment of chronic myeloid leukemia, and its efficacy and safety have been widely recognized, but there are still some adverse reactions. Among them, it is rare that the drug causes ovarian corpus luteum rupture and bleeding. In our clinical work, we just treated a patient with recurrent ovarian luteal cyst rupture and bleeding from imatinib, and followed up the whole course of the patient's pathogenesis, diagnosis and treatment effect. Therefore, this paper analyzes the clinical data and treatment of the case, in order to improve the clinical understanding and treatment of recurrent ovarian luteal cyst rupture and hemorrhage induced by imatinib. Methods: The clinical data and treatment of 1 case of recurrent ovarian corpus luteum cyst rupture bleeding from imatinib were analyzed, and the therapeutic effect was followed up. Results: Imatinib led to 6 times of corpus luteum rupture bleeding, which were successfully treated with conservative treatment. After taking Diane-35, the patient did not have corpus luteum rupture bleeding once again. And no adverse drug reactions. Conclusion: Imatinib can cause recurrent ovarian luteal cyst rupture and bleeding, conservative treatment is often effective. Diane-35 can effectively prevent and treat recurrent ovarian luteal cyst rupture and bleeding induced by imatinib. And the incidence of adverse drug reactions was low. But the specific mechanism that imatinib causes rupture of recurrent ovarian luteal cyst is unclear.
\end{abstract}

Keywords: Imatinib, Corpus Luteum Cyst, Treatment

\section{Research Background}

Rupture and hemorrhage of corpus luteum cyst is one of the common acute abdominal diseases in gynecology [1, 2], which occurs in the middle and late menstrual cycle, and is often seen in women of childbearing age, often under the inducement of exercise and sexual life. Clinically, the diagnosis of the disease mainly depends on laboratory and ultrasound examination [3]. After reviewing the literature and obstetrics and Gynecology textbooks, it is found that the reports of drug-induced rupture and hemorrhage of corpus luteum cyst are rare, and such cases are rarely encountered in clinical work. Chronic myeloid leukemia (CML) is a common hematologic disease, and is a chronic clonal myeloproliferative disorder with an incidence rate of 1-2/10 million $[4,5]$. Imatinib is the first-line drug in the treatment of chronic myeloid leukemia, and its efficacy and safety have been widely recognized [6-8]. However, imatinib still has 
some adverse reactions. Among them, it is rare that the drug causes ovarian corpus luteum rupture and hemorrhage $[9,10]$. Therefore, this paper analyzes the clinical data and treatment of a case of recurrent luteal cyst rupture and hemorrhage caused by imatinib, in order to improve the clinical understanding and treatment of this kind of disease.

\section{Methods}

\subsection{The Case Data}

A 17-year-old female student was unmarried and had no child; In June 2018, she was treated for "repeated fever". Bone marrow biopsy: Granulocyte proliferation was active, and no cluster distribution of precursor cells was found. It was easy to see in various stages of anaphase cells, eosinophils, decreased proliferation of erythrocytes, megakaryocyte proliferation, small megakaryocyte proliferation, and anterior tissue proliferation (MF-O). "CML" was considered. Clinical diagnosis: Chronic Myeloid Leukemia. After that, imatinib (imatinib mesylate capsule) was given orally $0.3 \mathrm{~g}$ once a day. The patient were treated with acute abdominal pain on November 12, 2018, December 13, 2018, September 21, 2019, March 8, 2020, April 2, 2020 and April 26, 2020, and each diagnosis was "rupture and hemorrhage of ovarian corpus luteum cyst". Main features: 1) onset time: 10-42 days after the last menstruation. 2) There was no obvious inducement for sudden acute lower abdominal pain. 3) Physical examination: obvious lower abdominal tenderness with or without muscle tension and rebound pain. 4) Gynecological color Doppler ultrasound: adnexal cystic mass, accompanied by pelvic ascites. The characteristics of the disease are shown in Table 1.

Table 1. Onset time and characteristics of color doppler ultrasound.

\begin{tabular}{llll}
\hline \multirow{2}{*}{$\begin{array}{l}\text { Onset time } \\
\text { (d) }\end{array}$} & \multicolumn{3}{l}{ characteristics of color doppler ultrasound } \\
\cline { 2 - 4 } & Location $(\mathbf{R} / \mathbf{L})$ & Size $(\mathbf{c m})$ & Depth of ascites $\mathbf{( c m )}$ \\
\hline 10 & R & $3.4 \times 2.8$ & 3.1 \\
18 & R/L & $2.7 \times 2.2 / 3.2 \times 2.0$ & 2.8 \\
12 & L & $7.1 \times 5.0$ & 2.7 \\
42 & L & $5.4 \times 3.4$ & 8 \\
20 & L & $5.9 \times 4.6$ & 1.7 \\
30 & L & $4.0 \times 3.9$ & 5.2 \\
\hline
\end{tabular}

Note: R: right-side adnexa. L: left-side adnexa.

Onset time: The time from the last menstruation.

\subsection{The Treatment}

Absolute bed rest, intravenous administration of Carbosulfan, hemocoagulase and other drugs to stop bleeding, pneumatic physiotherapy to prevent thrombosis, close observation of abdominal pain and abdominal signs, regular follow-up, gynecological ultrasound and other treatment were gaven to the patient. The patient recovered and discharged after 6 times of onset. In May 2020, Diane-35 (ethinylestradiol and cyproterone tablets) was given orally. Taking method: take it continuously for 21 days from the 2 nd to 5 th day of menstruation, and take it repeatedly.

\section{Results}

After oral administration of Diane-35, the patient had no symptoms of corpus luteum rupture, abdominal pain and other discomfort; Blood analysis and liver and kidney function were normal. In August 2020, gynecological color Doppler ultrasound showed that the uterus and bilateral adnexa were normal. The last follow-up time was December 2020. During the follow-up period, there were no adverse drug reactions related to Diane-35 and no other adverse drug reactions of imatinib.

\section{Discussion}

The ovaries form corpus luteum after ovulation. The normal mature corpus luteum is $2-3 \mathrm{~cm}$ in diameter, with an average diameter of $2.5 \mathrm{~cm}$. If it is not fertilized after ovulation, the luteal gland begins to atrophy on the 9th to 10th day after ovulation. The life span of luteal gland is usually about 2 weeks. When the corpus luteum continues to exist or there is a large amount of fluid in the cavity, making the diameter of the cavity more than $3 \mathrm{~cm}$, it is called corpus luteum cyst, and the severe cyst can reach $10 \mathrm{~cm}$ [11]. Corpus luteum cysts are large and distributed on the surface of ovary. Their texture is brittle. They are easy to cause rupture and bleeding under the inducement of sports and sexual life [12]. Rupture of corpus luteum cyst can occur in married or unmarried women, especially in women of child-bearing age. Generally in the 20 th to 27 th day of menstrual cycle; The main clinical manifestation was sudden lower abdominal pain with or without nausea, vomiting, frequent urination and defecation. There are also a small number of patients with abdominal pain occurred in the middle of the menstrual cycle or the 30th to 40th day of the menstrual cycle. Severe cases can appear dry mouth, palpitations, dizziness, dizziness, fainting and other shock symptoms.

Chronic myeloid leukemia (CML) is a myeloproliferative tumor driven by the BCR-ABL fusion gene encoding BCR-ABL protein with a sustained tyrosine kinase activity. The incidence rate increases with age. The expression of BCR-ABL gene can activate many signal transduction pathways in CML, and mediate cell proliferation and survival. Imatinib, a tyrosine kinase inhibitor (TKIs), is the main drug for the treatment of CML. Clinical findings show that imatinib is generally well tolerated, and its grade 3 and grade 4 non hematologic toxicity are not common [13]. At present, imatinib is the first choice for the treatment of CML, its efficacy and safety are widely recognized. But there are still some adverse reactions [7-10]. Among them, repeated rupture and hemorrhage of ovarian corpus luteum cyst caused by this drug is very rare. In this study, the patient had never had ovarian corpus luteum cyst rupture and bleeding before taking imatinib. Since taking imatinib, ovarian corpus luteum cyst rupture and bleeding occurred repeatedly, and the rare complication of ovarian corpus luteum cyst rupture caused by imatinib was identified in the drug instructions, It shows that the rupture and bleeding of luteal cyst is closely 
related to imatinib. However, the mechanism of upture and hemorrhage of ovarian corpus luteum cyst caused by this drug is still unclear. From the mechanism of the formation of ovarian luteal cyst, imatinib may lead to the delayed atrophy of the luteal body, increase the secretion of the luteal fluid, increase the fragility of the wall of the cyst, and lead to the more likely rupture and bleeding of the luteal cyst. Therefore, if female patients of childbearing age have acute lower abdominal pain during taking imatinib, the possibility of rupture and bleeding of corpus luteum cyst should be considered, and gynecological color Doppler ultrasound examination should be improved in time to avoid missed diagnosis.

The treatment of ovarian corpus luteum cyst rupture bleeding can choose conservative treatment, sach as mainly in bed rest, using hemostatic drugs to stop bleeding, preventing thrombosis and other treatment. The symptoms of abdominal pain and abdominal signs were closely observed, and gynecological color Doppler ultrasound was followed up. If the condition worsened, surgery was performed if necessary. Xiayun Liu's research on 32 patients with conservative treatment, were successful. In this study, the patient had 6 times of ovarian corpus luteum cyst rupture and bleeding, all of which were successfully treated with conservative treatment.

Ovarian luteal cyst is a physiological cyst formed after ovulation, which is closely related to ovarian cycle. Therefore, to prevent the recurrence of the disease, we can choose standard oral short acting contraceptives to inhibit ovulation. Diane-35 (ethinylestradiol and cyproterone tablets) is a compound short acting oral contraceptive. Its main components are ethinylestradiol and cyproterone, which can effectively inhibit ovulation. And the drug safety is high [15, 16]. In this study, the patient took Diane-35 orally for 7 cycles, the patient did not have ovarian cyst rupture and bleeding again, and the follow-up gynecological ultrasound showed that bilateral adnexal cyst disappeared, and there was no adverse drug reaction of Diane-35. These results indicate that Diane-35 can effectively prevent and treat the repeated rupture and hemorrhage of ovarian corpus luteum cyst caused by imatinib.

\section{Conclusion}

In conclusion, imatinib can lead to repeated rupture and hemorrhage of ovarian luteal cyst, and conservative treatment is often effective. Diane-35 can effectively prevent and treat repeated ovarian corpus luteum cyst rupture and hemorrhage caused by imatinib, and the incidence of adverse drug reactions was low. But the specific mechanism of repeated ovarian corpus luteum cyst rupture caused by imatinib is still unclear. The mechanism may be related to imatinib induced delayed corpus luteum atrophy, increased secretion of corpus luteum fluid and increased fragility of corpus luteum cyst wall. In addition, there are few cases in this study, and more clinical data are needed to support the results.

\section{Funding}

This work was supported by grants from the Scientific Research Support Program for Postdoctoral of Jiangsu Province (2019K064), the Major Science and Technology Program of Changzhou Health and Family Planning Commission (ZD201812), the Scientific Research Support Program for "333 Project" of Jiangsu Province (BRA2019161).

\section{References}

[1] Xing Xie, Beihua Kong, Tao Duan, et al. Obstetrics and Gynecology ninth edition [M]. Beijing: People's Health Publishing House, 2018: 77-78.

[2] Hui Liu, Hong Xiang, Ruixue Mu, et al. Value of transvaginal two-dimensional contrast-enhanced ultrasonography in diagnosing atypical ovarian corpus luteum hematoma $[\mathrm{J}]$ BioMed Research International, 2018, 2018 (5): 1-11.

[3] Huiqing Wang, Jie Zhan. Ultrasonographic features and clinical analysis of rupture of corpus luteum cyst [J]. Journal of Medical Imaging, 2020, 30 (6): 116-118.

[4] HUANG X, CORTES J, KANTARJIAN H. Estimations of the increasing prevalence and plateau prevalence of chronic myeloid leukemia in the era of tyrosine kinase inhibitor therapy [J]. Cancer, 2012, 118 (12): 3123-3127.

[5] Yanzhi Zhang, Ling Qin. A case of chronic myeloid leukemia with myelofibrosis in children [J]. Hainan Medical Journal, 2021, 32 (8): 1074-1075.

[6] Gugliotta G, Castagnetti F, Apolinari MA, et al. First-Line treatment of newly diagnosed elderly patients with chronic myeloid leukemia: current and emerging strategies [J]. Drugs, 2014, 74 (6): 627-643.

[7] Fei Li, Xiaojie Zhang, Rongyan Zhang, et al. Imatinib for the first-line treatment of chronic myeloid leukemia in chronic phase: a single center retrospective analysis of 10 years $[\mathrm{J}]$. Chinese Journal of Clinical Oncology, 2016, 43 (10): 432-437.

[8] Kagimoto Y, Mizuashi M, Kikuchi K, et al. Lichenoid drug eruption with hyperpigmentation caused by imatinib mesylate [J]. International Journal of Dermatology, 2014, 53 (3): e161e162.

[9] Hongmei Zhu. Literature analysis of 224 cases of imatinib mesylate adverse reactions [J]. Chinese Journal of Pharmacovigilance, 2018, 15 (12): 733-737.

[10] Jun Ma, Jianmin Luo, Yan Wen, et al. Clinical study of domestic imatinib mesylate in the treatment of newly diagnosed chronic myeloid leukemia in chronic phase [J]. Journal of Clinical Hematology, 2018, 31 (11): 841-845.

[11] Xiaolan He, Hong Gao, Shiyi Liu. Study on the differential diagnosis of ovarian corpus luteum rupture and ectopic pregnancy rupture by transvaginal color Doppler ultrasound $[\mathrm{J}]$ Journal of North Sichuan Medical College, 2019, 34 (5): 600-603.

[12] Hua-Di Yang, Meng-Ting Xia, Yi-Feng Shao, et al. Recurrent ovarian hemorrhage in a patient with aplastic anemia: a case report [J]. Medicine, 2018, 97 (27): 11409-11414. 
[13] Fengjiao Fan, Jixiang He, zhangkun Li, Yirong Jiang. A case of drug-induced lung injury induced by imatinib [J]. Journal of Clinical Internal Medicine, 2021, 38 (4): 282-283.

[14] Xiayun Liu, Yanna Lin. Clinical analysis of 60 cases of rupture and hemorrhage of ovarian corpus luteum cyst [J]. Shenzhen Journal of Integrated Traditional Chinese and Western Medicine, 2017, 27 (23): 109-110.
[15] Xing Xie, Beihua Kong, Tao Duan, et al. Obstetrics and Gynecology ninth edition [M]. Beijing: People's Health Publishing House, 2018: 369-370.

[16] Ling Qin. Diane 35 combined with metformin in the treatment of polycystic ovary syndrome [J]. Journal of practical gynecological endocrinology, 2019, 24 (6): 78-78. 